\title{
The new W7-X Logbook - A Software for Effective Experiment Documentation and Collaborative Research at Wendelstein 7-X
}

\author{
M. Grahl, A. Spring, T. Bluhm, H.-S. Bosch, R. Brakel, S. Dumke, P. van Eeten, M. Grün, M. Hirsch, A. \\ Holtz, H. Laqua, M. Lewerentz, H. Riemann, A. Werner and the W7-X Team \\ Max-Planck-Institut für Plasmaphysik, \\ Wendelsteinstraße 1, 17491 Greifswald, Germany
}

\begin{abstract}
Wendelstein 7-X (W7-X) completed its second operation phase in December 2017. A large number of diagnostics were operated in nearly 1000 experiment programs (XP) by an international research team. For the documentation of W7-X XPs, a new electronic logbook software has been developed. The software has been designed for the needs of W7-X researchers and engineers: a web-based logbook application for the entire team. For an effective documentation, large parts of the logbook content comes from automatically generated logs, complemented by the team members via web browser or REST API. The W7-X control software generates log entries for an XP during program execution. This includes automatic extraction of configuration information from the planned program, which is later represented as searchable tags within the logbook. In addition, users can add supplementary tags and rich-text comments to all logs. The logbook allows full-text search and range queries for numeric values: both with millisecond response times. A dedicated web page for each XP log contains overview plots from measurement data, generated on the fly from archived data. Separate component logs are created in the same way for diagnostics and machine sub-systems for different use cases, e.g., XPs, standalone tests, or calibrations. The logbook hosts separate pages for device operation and the W7-X components, providing status information and additional features. In this way, it effectively supports the operation of W7-X.
\end{abstract}

\section{Introduction}

The stellarator experiment Wendelstein 7-X (W7-X) [1] completed the first part of its second operation phase (OP 2.1a) in December 2017. Even in these early phases, nearly 40 diagnostic systems were already operated by an international research team [2]. In the first operational phases - with discharges limited to $100 \mathrm{~s}$ - up to 50 experiment programs (XP) can be conducted per day. Operating in a typical week with two or three experiment days, the number of programs sums up to $1000-1500$ XPs per operational phase, which are dedicated to a broad range of research topics. The CoDaC software chain [3] extensively supports these activities. For the documentation of W7-X operation and the collective utilization of experiment data, a new electronic logbook software has been developed and used for the first time in OP1.2a.

\section{Electronic logbooks}

Electronic logbooks play an important role in many engineering and scientific fields. Different implementations of electronic logbooks were already used at other fusion experiments, e.g. at the tokamaks Alcator C-Mod [4] and TEXTOR [5]. In contrast to these implementations, the $\mathrm{W}-\mathrm{X}$ logbook has been designed as full-featured web service and is not limited to experiment logs and comments by the device operator. Logs in the W7-X logbook can be created coherently for plasma discharges as well was for commissioning or calibration runs of diagnostics and subsystems. Rich-text comments can be added by all members of the W7-X team. In addition, the logbook supports standardized metadata as basis for a fast search index. This approach is comparable with other proposed solutions [6]. In the W7-X logbook, the meta information is extracted automatically from W7-X control system data. In this way, the W7-X logbook provides an excellent approach for documentation of and access to W7-X experiment data. It accelerates experiment evaluation and information exchange by connecting all available experiment data including team comments and data plots on an interactive website in a comprehensive system.

\section{Basic concepts}

The implementation of the W7-X logbook has been focused on good usability and the automatic creation of content in a standardized form. Combining advanced search options and a seamless integration into the CoDaC software stack, the logbook provides a system for highly productive research for the entire W7-X team. 


\subsection{Central logbook for the W7-X team}

Research at W7-X is done in collaboration with a large team of engineers and physicists. The W7-X logbook provides dedicated views and adaptive features for different user roles, e.g., engineer in charge, session leader, diagnostic team, and component operators. Every W7-X team member can add information to the logbook, which requires features like version control and a flexible user rights management.

\subsection{Automatic data enrichment}

The data acquisition systems of the W7-X subsystems and diagnostics produce a large amount of data for each discharge, which will sum up to petabytes per year in the future. For an effective analysis, a high-level description of this mass data is needed, as well as journals of experiment runs and other activities. As manually input tends to be time consuming and error-prone, all programmatically extractable information has to be generated automatically. In addition, manually user input needs support by a modern user interface (Fig. 1). For the main content of the logbook, the W7-X experiment planning and control software (Xedit \& Xcontrol) automatically creates logs. Metadata of an experiment program is obtained from its determining parameters at planning time using the Component Model framework [7] as part of the experiment program editor [8]. This includes key parameters such as the intended plasma heating, fuelling, magnetic field configuration and main diagnostic settings. During the experiment run, this metadata is logged to the archive together with the experiment sequence and more planning information retrieved from the W7$\mathrm{X}$ proposal database. These automatic logs provide the basis for each experiment entry in the logbook. The W7-X team members complete these logs by adding additional information, like a result flag or other evaluating metadata. This happens manually in a web browser or programmatically via script or software.

\subsection{Tags for standardized input}

The logbook supports different kinds of input for notes, remarks, and comments. Next to images and free-form text, key parameters are included as tags, which are shown as simple labels on the logbook website (Fig. 2). These tags are defined as structured objects within the logbook system. The value of a tag can be a string or a numeric value. For numeric tags, an attribute for the physical unit can be set. In addition, a fixed list with potential values can be defined, e.g. available gas types for gas injection. Tags are grouped in categories: experiment (containing tags like session leader, proposal number, and program type), heating, fuelling, magnetic field, and diagnostics. Tag templates are defined and managed in an extendable tag catalogue. On the logbook website, the user can select one or more tags from the catalogue and add them to a log in a convenient manner. The standardized information input leads to better search results and is crucial when logbook data is used for an automatic analysis of W7-X experiments.

\subsection{Fast search index}

The logbook is implemented as a three-tier architecture with presentation layer (a web interface), middle tier, and persistence layer. In the backend, the logbook data is eventually stored in the W7-X archive. The middle tier implementation includes a fast search index based on elasticsearch [9]. It is used for providing full-text search for the complete logbook content with a near real time response time. In addition, the standardized tags allow searches in numeric ranges using a simple syntax. For example a search query for all experiments with a microwave heating duration between 20 and 60 seconds would be: id:XP_* AND tags.ECRH। duration:[20 TO 60]

The flexible data model of the search index leads to a high extensibility of the software. Extensibility is an important factor for working with scientific data models, as requirements can change rather quickly and disruptively, e.g., caused by new results or research approaches.

\subsection{RESTful web service}

The logbook has to allow an easy integration of logbook content into other CoDaC tools, as well as the usage for automatic data analysis. Therefore, it has been implemented as a RESTful web service [10], using standard protocols: HTTP (hypertext transport protocol), JSON (JavaScript object notation) and HTML (hypertext markup language). In a RESTful interface, the data is represented as resources on a server, which can be addressed by a URL (uniform resource locator). A small number of operations are used to interact with these resources, while connections between resources are represented via hyperlinks. This leads to a standardized interface, which is accessible in any modern programming language.

\section{Application}

Implemented as a central collaboration tool, the logbook provides dedicated views to meet different use cases: experiment overview, component and diagnostic specific documentation, physicists' and engineers' view.

\subsection{Overview listing}


The overview listing provides a streamlined interface in the style of well-known web search engines and acts as the starting point for most use cases of the logbook. Next to the single input field for all kinds of search requests, a calendar widget and quick links to the last experiment days provide fast access to experiment logs sorted by date. The resulting listing shows hyperlinks to experiment log pages and other resources, enriched with classifying tags and user comments for a fast overview.

\subsection{Experiment log website}

The log of each experiment program conducted by the W7-X segment control gets its own webpage with a unique web address immediately at the start of the experiment run. A second version of the log is written on the end of the program, updating the log. The website contains the central information of an experiment, such as the XP number, start and end time, name, description, and technical execution status. The logbook provides version control for the log entries, so users can see the history of a log with all changes.

A typical XP log page is shown in Fig. 3. For a better overview, general tags are displayed on the XP log level, while component specific tags are added to the associated component logs. Examples for experiment related tags are the configuration of the magnet system, power and duration of the heating systems: electron cyclotron resonance heating and neutral beam injection. For the fuelling, gas type and timing are shown for gas and pellet injection systems, as well as tags for injective diagnostics, which are used for transport and impurity studies. Session details, such as experiment leader, program type, corresponding proposals, and a result flag, are also included as tags. The users can add new tags from the tag catalogue directly on the website. The component logs, with user comments concerning the component status and component specific tags, are listed in editable form on the XP website. The integrated text-editor allows text formatting and the integration of images. In this way, users can share their components settings, notes and observations in a fast and easy manner with the W7-X team.

\subsection{Interactive preview plots}

An important part of experiment execution is a fast evaluation of the experiment success, for example for planning the next steps of a session. The logbook supports this with live plots from data of central systems and diagnostic measurements. The website of an experiment log provides a fast preview with overview plots showing the main plasma properties of the discharge (Fig. 4). The preview plots are available right after, or even during, a discharge, as they are generated on the fly with JavaScript from the web service of the W7-X data archive [11]. The central W7-X archive contains the raw and analysed data from the diagnostic systems, as well as machine operation data. By using a server side downscaling the data is loaded and displayed in a timely manner, even for data from high-resolution data acquisition systems. The configurable plots are able to show multiple data streams in a row. The user can freely configure the plots by selecting any data available from the archive, or use one of the predefined presets. The plots provide interactive features like zooming and panning using the mouse.

\subsection{Component pages}

Next to the central logs for W7-X experiment programs, the logbook provides dedicated areas for the machine subsystems, heating and fuelling systems and diagnostics. The component overview page on the logbook website currently lists over 80 systems. The logbook provides special status overviews of different system groups e.g., machine subsystems, or profile / scrap-off layer diagnostics. Every system has its own logbook area on the website (Fig. 5). This includes a start page containing general information about the system, e.g., team members, hardware, and data access links. In addition, the website provides the option to view and edit all corresponding logs of a component: for XPs, as well as stand-alone runs and tests. Logs can be accessed by selection from a calendar tool or from a listing of all items.

\subsection{Engineer in charge logbook}

The logbook provides a special area for the W7-X device operation. The engineer in charge uses the logbook for shift logs and the documentation of planned activities and incidents concerning the commissioning and operation of the machine and all peripheral systems. For a technical documentation of W7-X operation, the logbook provides daily reports, which include overviews of experiment programs and machine operation events.

\section{Implementation}

The logbook was implemented iteratively in an agile software development process, starting from a small proof-ofprinciple prototype. The requirements were derived mainly from experiences from the first campaigns. Further requirements were collected from $W 7-X$ team members, representing different roles in the project.

For the server part of the software, a RESTful web service has been written in Java, providing resources in JSON or HTML format. The logbook offers its features as interactive websites for the graphical user interface. In addition, the resources are available as JSON for programmatic access. Elasticsearch is used for providing the search index as well as 
version control. The logbook website uses a single sign-on mechanism, so the user is automatically authenticated on a Windows machine. Users and teams are managed within the central user database of the institute.

\section{Conclusion}

A central web based logbook software has been implemented for the use by the entire W7-X team. The basis of the content is generated automatically by the W7-X control software. The logbook includes special areas for the logs of the diagnostics and subsystems of W7-X, as well as for the engineer in charge. Fast full-text search and range-based queries with tags are important for manual exploration of the results, as well as for automatic analysis. All functions are available on a website or via RESTful web service. The logbook allows rich-text user comments and provides an interactive website with plots generated on the fly from recent data. The logbook was quickly adopted by the W7-X team members. It is appreciated as a crucial tool for effective experimentation and acts as the starting point for the exploration and analysis of experiment data at W7-X.

\section{Acknowledgments}

The authors would like to thank all users of the logbook and esp. those who helped collecting requirements and gave very helpful feedback and bug reports: U. Höfel, A. von Stechow, H. Thomsen, H. Neilson, N. Pablant, S. Lazerson, the W7-X session leaders, and many more.

This work has been carried out within the framework of the EUROfusion Consortium and has received funding from the Euratom research and training programme 2014-2018 under grant agreement No 633053. The views and opinions expressed herein do not necessarily reflect those of the European Commission.

\section{References}

[1] H.-S. Bosch, et al. "Final integration, commissioning and start of the Wendelstein 7-X stellarator operation", Nucl. Fusion 57, 116015 (2017)

[2] R.C. Wolf, et al. "Major results from the first plasma campaign of the Wendelstein 7-X stellarator", Nucl. Fusion 57, 102020 (2017)

[3] A. Spring, et al. "Establishing the Wendelstein 7-X steady state plasma control and data acquisition system during the first operation phase", Fusion Eng. Des., 123 (2017), pp. 579-583.

[4] T.W. Fredian, et al. "Web based electronic logbook and experiment run database viewer for Alcator C-Mod", Fusion Eng. Des., 81 (2006), pp. 1963-1967.

[5] A. Krämer-Flecken, et al. "Experiment management system - a way towards a transparent Tokamak", Fusion Eng. Des., 83 (2008), pp. 375-381.

[6] J.A. Stillerman, et al. "Data catalog project-A browsable, searchable, metadata system", Fusion Eng. Des., 112 (2016), pp. $995-$ 998.

[7] M. Lewerentz, et al. "Experiment planning using high-level component models at W7-X", Fusion Eng. Des., 87 (2012), pp. 19491953.

[8] A. Spring, et al. "A W7-X experiment program editor-A usage driven development", Fusion Eng. Des., 87 (2012), pp. 19541957.

[9] https://www.elastic.co/products/elasticsearch, [Online], Accessed: Sep. 2018

[10] R. Fielding "Architectural Styles and the Design of Network-based Software Architectures", Dissertation, University of California - Irvine (2000)

[11] M. Grahl, et al. "Archive WEB API: A web service for the experiment data archive of Wendelstein 7-X", Fusion Eng. Des., 123 (2017), pp. 1015-1019. 


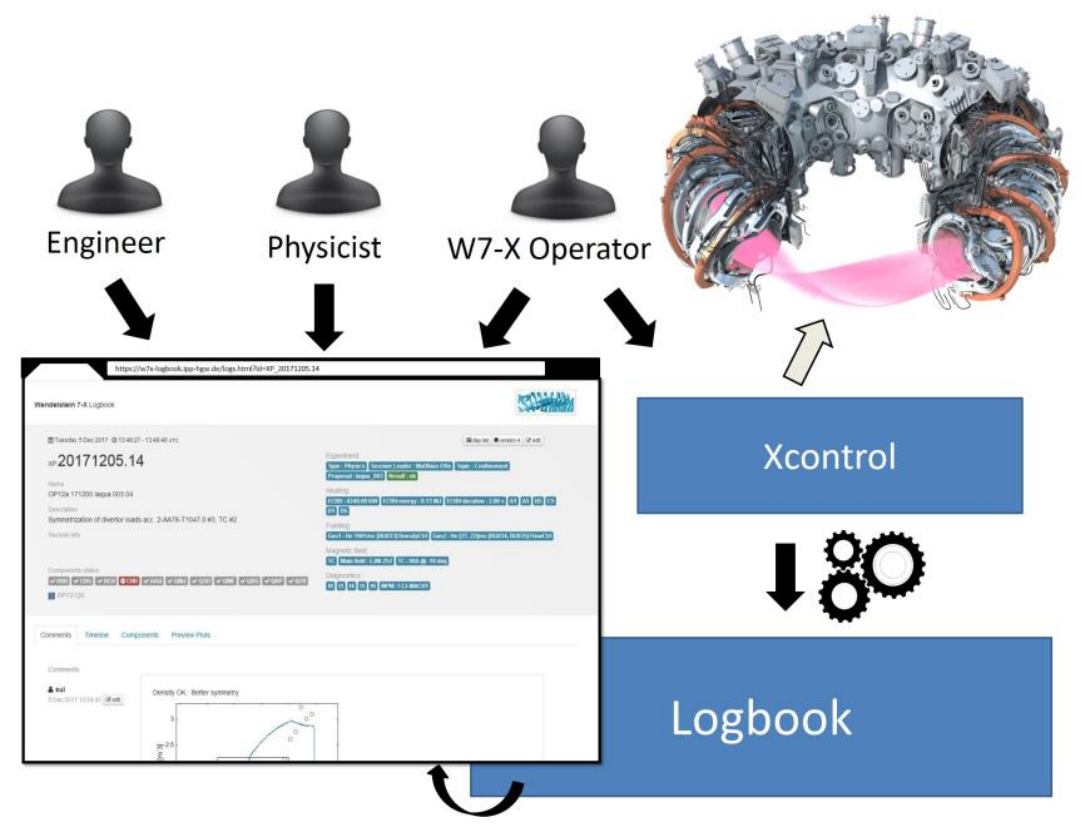

Fig. 1 - Logbook content is created by the W7-X control software and complemented by W7-X team members.

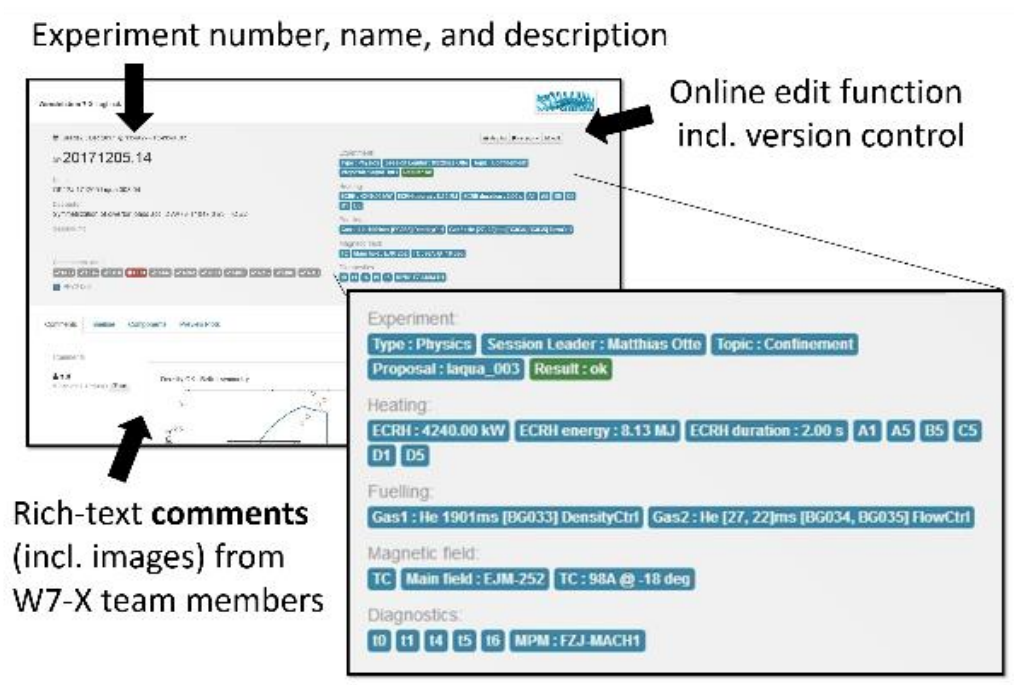

Tags: Key experiment parameters for searches and categorization

Fig. 2 - Key parameters are shown as tags on the experiment website. 


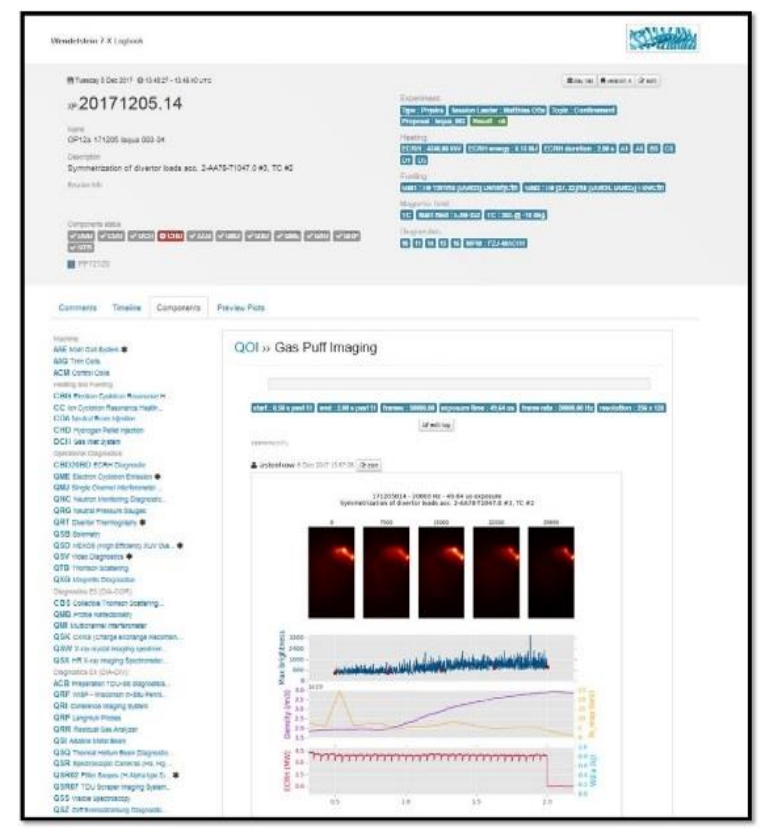

Fig. 3 - Experiment program log with tags (top right) and the corresponding component logs (bottom). Systems with comments are marked in the listing with an asterisk.

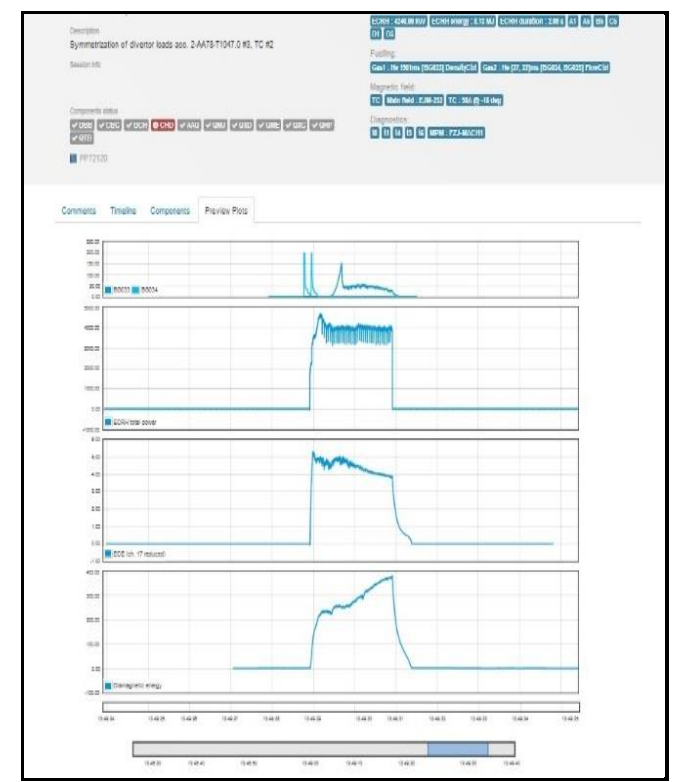

Fig. 4 - Integrated preview plots show the key results on the logbook XP page. The interactive, customable plots are generated on the fly from archived data. 


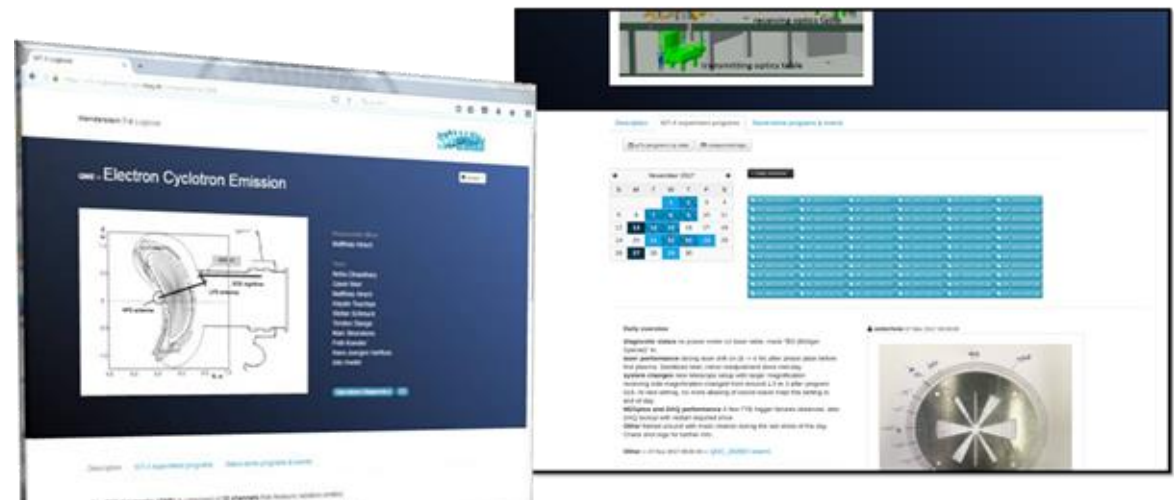

Fig. 5 - Component pages: each W7-X subsystem or diagnostic has its own area on the logbook website. 